\title{
Spectrofluorometric Determination of Certain Antihyperlipidemic Agents in Bulk and Pharmaceutical Preparations
}

\author{
Ramzia I. El-Bagary, ${ }^{1}$ Ehab F. ElKady, ${ }^{1}$ and Ahmed M. Kadry ${ }^{2}$ \\ ${ }^{1}$ Pharmaceutical Chemistry Department, Faculty of Pharmacy, Cairo University, Cairo 11562, Egypt \\ ${ }^{2}$ National Organization for Drug Control and Research (NODCAR), P.O. Box 29, Giza 35521, Egypt \\ Correspondence should be addressed to Ahmed M. Kadry, dr.ahmedkadry@ gmail.com
}

Copyright (C) 2012 Ramzia I. El-Bagary et al. This is an open access article distributed under the Creative Commons Attribution License, which permits unrestricted use, distribution, and reproduction in any medium, provided the original work is properly cited.

\begin{abstract}
A simple, rapid, and sensitive spectrofluorometric method was developed for the determination of three antihyperlipidemic drugs, namely, rosuvastatin calcium (RSV), ezetimibe (EZE), and pitavastatin calcium (PIT). The method is based on measuring the native fluorescence of the cited drugs at their optimum excitation and emission wavelengths. The fluorescence intensity was measured at $\lambda$ em $362 \mathrm{~nm}, 309 \mathrm{~nm}$, and $373 \mathrm{~nm}$ upon excitation at $\lambda$ ex $315 \mathrm{~nm}, 260 \mathrm{~nm}$, and $245 \mathrm{~nm}$ for RSV, EZE, and PIT, respectively. The calibration graphs were linear over the concentration ranges 0.50-10.0, 0.25-4.0, and $0.10-3.00 \mu \mathrm{g} \mathrm{mL}^{-1}$ for RSV, EZE, and PIT, respectively. Besides, a spectrofluorometric method for the simultaneous determination of RSV and EZE was developed. The fluorescence was measured at $\lambda \mathrm{em} 309 \mathrm{~nm}$ for EZE and $432 \mathrm{~nm}$ for RSV upon excitation at $\lambda$ ex $260 \mathrm{~nm}$ for both. The proposed methods were applied to the determination of the cited drugs either in bulk and pharmaceutical preparations.
\end{abstract}

Keywords: Spectrofluorometry, rosuvastatin calcium, ezetimibe, pitavastatin calcium, native fluorescence, pharmaceutical preparation

\section{Introduction}

Rosuvastatin calcium (RSV), [(E)-7-\{4(4-Fluorophenyl)-6-isopropyl-2-[methyl (methylsulfonyl) amino] pyrimidin-5-yl\}-3,5-dihydroxyhept-6-enoic acid calcium salt], ezetimibe (EZE), [1-(4fluorophenyl)-3-[3-(4-fluorophenyl)-3-hydroxy-propyl]-4-(4-hydroxyphenyl)-azetidin-2-one], and pitavastatin calcium (PIT), [(+)-monocalcium bis $\{(3 \mathrm{R}, 5 \mathrm{~S}, 6 \mathrm{E})-7-[2-c y c l o p r o p y l-4-(4-f l u o r o p h e n y l)-3-$ quinolyl]-3,5-dihydroxy-6-heptenoate $\}$ ] are novel antihyperlipidemic oral drugs. Chemical structure of RSV, EZE, and PIT is shown in Figure 1. RSV and PIT are members of a class of statins that lower cholesterol levels by inhibiting the enzyme HMG-CoA reductase; this enzyme catalyzes the conversion of (HMG-CoA) to mevalonate, an early and rate-limiting step in cholesterol biosynthesis $[1,2]$. EZE is a drug that lowers cholesterol by preventing the absorption of cholesterol from dietary and biliary sources through blocking the transport of cholesterol through the intestinal wall [3]. EZE, coadministered with statins, is licensed for the treatment of primary hypercholesterolemia in patients, 
<smiles>CC(C)c1nc(N(C)S(C)(=O)=O)nc(-c2ccc(F)cc2)c1/C=C/[C@@H](O)C[C@@H](O)CC(=O)[O-]</smiles>

(a)<smiles>O=C1[C@H](CC[C@H](O)c2ccc(F)cc2)[C@@H](c2ccc(O)cc2)N1c1ccc(F)cc1</smiles>

(b)<smiles>O=C([O-])C[C@@H](O)C[C@@H](O)/C=C/c1c(CC2CC2)nc2ccccc2c1-c1ccc(F)cc1</smiles>

(c)

Figure 1: Chemical structures of rosuvastatin calcium (a), ezetimibe (b), and pitavastatin calcium (c).

poorly controlled with a statin alone, and for homozygous familial hypercholesterolemia [4]. A detailed survey of analytical literature for RSV revealed several methods based on varied techniques, HPLC [5-7], spectrophotometry, [8] and high-performance thin-layer chromatography [9]. Similarly, a survey of analytical literature for the determination of EZE either in biological samples or pharmaceutical preparations revealed methods based on HPLC [10-13] and LC/MS/MS [14, 15] for its determination in human plasma, UV-spectrophotometric determination of combination with other drug [16] and stabilityindicating LC $[17,18]$. Besides, different analytical methods for the determination of PIT have been reported, including spectrophotometry [19], high-performance thin-layer chromatography [20], and LC/MS/MS for its determination in human plasma [21-24].

Spectrofluorometry has long been applied in the field of pharmaceutical analysis of many drugs [25-28] because of the higher sensitivity than is attainable in absorption spectrophotometry. A necessary condition for a compound to fluoresce is that it absorbs light in the UV or visible region of the spectrum. Accordingly, compounds that have a conjugated $\pi$-electron system may give efficient reemission of the absorbed energy as a direct method for the determination in which the native fluorescence of the molecule is measured [25-28]. The objective of this work was to develop sensitive, simple, precise, and rapid spectrofluorometric methods of analysis for PIT, RSV, and EZE in bulk and in their pharmaceutical preparations and for the combination drug product that contains RSV and EZE. 


\section{Experimental}

\subsection{Instrumentation}

A Shimadzu RF-1501 spectrofluorimeter (Japan) and Soniclean sonicator-degasser (Australia) were used.

\subsection{Reagents and Reference Samples}

Pharmaceutical grade RSV (certified to contain 99.91\%) and Crestor $5 \mathrm{mg}$ tablets nominally containing $5.217 \mathrm{mg}$ of RSV per tablet (batch no. 100268) were supplied by AstraZeneca-Egypt. EZE (certified to contain $99.93 \%$ ) and Ezetrol $10 \mathrm{mg}$ tablets nominally containing $10.0 \mathrm{mg}$ of EZE per tablet (batch no. 303345) were supplied by Merck Sharp \& Dohme-Egypt. PIT (certified to contain 99.80\%) and Livalo $2 \mathrm{mg}$ tablets nominally containing $2.09 \mathrm{mg}$ PIT per tablet (batch no. 3079200) were supplied by LillyUSA. Rosuvas EZ10 mg tablets nominally containing $10.0 \mathrm{mg}$ of EZE and $10.434 \mathrm{mg}$ of RSV per tablet (batch no. 2220206) were supplied by Ranbaxy-India. Methanol HPLC grade and acetonitrile HPLC grade were purchased from Sigma-Aldrich, Germany.

\subsection{Standard and Test Solutions}

\subsubsection{Preparation of Standard Solutions}

Standard stock solutions of $100 \mu \mathrm{g} \mathrm{mL}^{-1}$ of each drug were prepared in methanol as selected solvent for RSV and PIT and in acetonitrile as selected solvent for EZE.

\subsubsection{Preparation of Test Solutions}

For Pharmaceutical Preparations Containing RSV, EZE, and PIT Alone

Twenty tablets of each of crestor $5 \mathrm{mg}$, ezetrol $10 \mathrm{mg}$, and livalo $2 \mathrm{mg}$ tablets were separately weighed and finely powdered in a mortar. A tablet powder equivalent to $10 \mathrm{mg}$ of each drug (RSV-free, EZEand PIT-free) was accurately weighed and separately transferred to three $100 \mathrm{~mL}$ volumetric flasks. The tablet powder of each drug was dissolved using methanol as selected solvent for RSV and PIT and acetonitrile as selected solvent for EZE, then sonicated for 15 minutes, and completed to volume with the selected solvent. The solutions were then filtered through $0.45 \mu \mathrm{m}$ nylon syringe filter, followed by serial dilution to required concentrations for each experiment.

\section{For Rosuvas-EZ Tablets Containing Binary Mixture RSV and EZE}

Twenty tablets of rosuvas ez $10 \mathrm{mg}$ were weighed and finely powdered in a mortar. A tablet powder equivalent to $10 \mathrm{mg}$ of RSV-free and equivalent to $10 \mathrm{mg}$ of EZE was accurately weighed and transferred to a $100 \mathrm{~mL}$ volumetric flask. The tablet powder was dissolved using acetonitrile as selected solvent, then sonicated for 15 mins, and made up to the mark with the selected solvent. The solution was then filtered 
through $0.45 \mu \mathrm{m}$ nylon syringe filter, followed by serial dilution to the required concentrations for each experiment.

\subsection{General Procedures and Calibrations}

\subsubsection{For RSV, EZE, and PIT Alone}

Accurately measured aliquots of working standard solutions equivalent to $5.0-100.0 \mu \mathrm{g}, 2.5-40.0 \mu \mathrm{g}$ and 1.0-30.0 $\mu \mathrm{g} \mathrm{RSV}$, EZE and PIT were accurately measured and separately transferred from their stock solutions into three sets of $10 \mathrm{~mL}$ volumetric flasks; then each flask was completed to volume with the selected solvent of each drug. The native fluorescence intensities were measured at the specified excitation and emission wavelengths for each drug ( $\lambda$ ex $315 \mathrm{~nm}-\lambda$ em $362 \mathrm{~nm}$ ), ( $\lambda$ ex $260 \mathrm{~nm}-\lambda$ em $309 \mathrm{~nm}$ ), and ( $\lambda$ ex $245 \mathrm{~nm}-\lambda$ em $373 \mathrm{~nm}$ ) for RSV, EZE, and PIT, respectively. A calibration curve was obtained for each drug by plotting fluorescence intensity $(\mathrm{F})$ against concentration $(\mathrm{C})$, and the regression parameters were computed for each drug.

\subsubsection{For RSV-EZE Laboratory-Prepared Mixtures}

Different aliquots from RSV and EZE stock solutions equivalent to 4.0-100.0 $\mu \mathrm{g}$ and 2.0-50.0 $\mu \mathrm{g}$, respectively, were accurately measured and transferred into a set of $10 \mathrm{~mL}$ volumetric flasks, and the volumes were completed with acetonitrile. The native fluorescence intensity was measured at $\lambda \mathrm{em}$ $432 \mathrm{~nm}$ for RSV and $309 \mathrm{~nm}$ for EZE upon excitation at $\lambda$ ex $260 \mathrm{~nm}$ for both drugs, then plotted against corresponding concentration, and the regression parameters were computed.

\section{Results and Discussion}

Literature survey reveals that only liquid chromatographic and spectrophotometric methods have been reported for the determination of RSV, EZE, and PIT. Thus, the development of spectrofluorometric methods for the determination of the cited drugs in the bulk or pharmaceutical preparations was of interest as no such methods have been reported for the three drugs. The proposed spectrofluorometric methods were successfully applied to estimate RSV, EZE, PIT and the binary mixture RSV-EZE in tablet dosage forms as no interference of excipients was found (Table 1).

\subsection{Method Development}

\subsubsection{For RSV, EZE, and PIT Alone}

Selection of excitation wavelengths for RSV, EZE, and PIT was based on the maximum wavelength of absorption in the UV region; then excitation spectra are determined by measuring the emission intensity at fixed wavelength while varying the excitation wavelength for each of the cited drugs. Optimum excitation wavelengths found for RSV, EZE, and PIT are $315 \mathrm{~nm}, 260 \mathrm{~nm}$, and $245 \mathrm{~nm}$, respectively. Also, selection of suitable emission wavelength was done by scanning the emission 


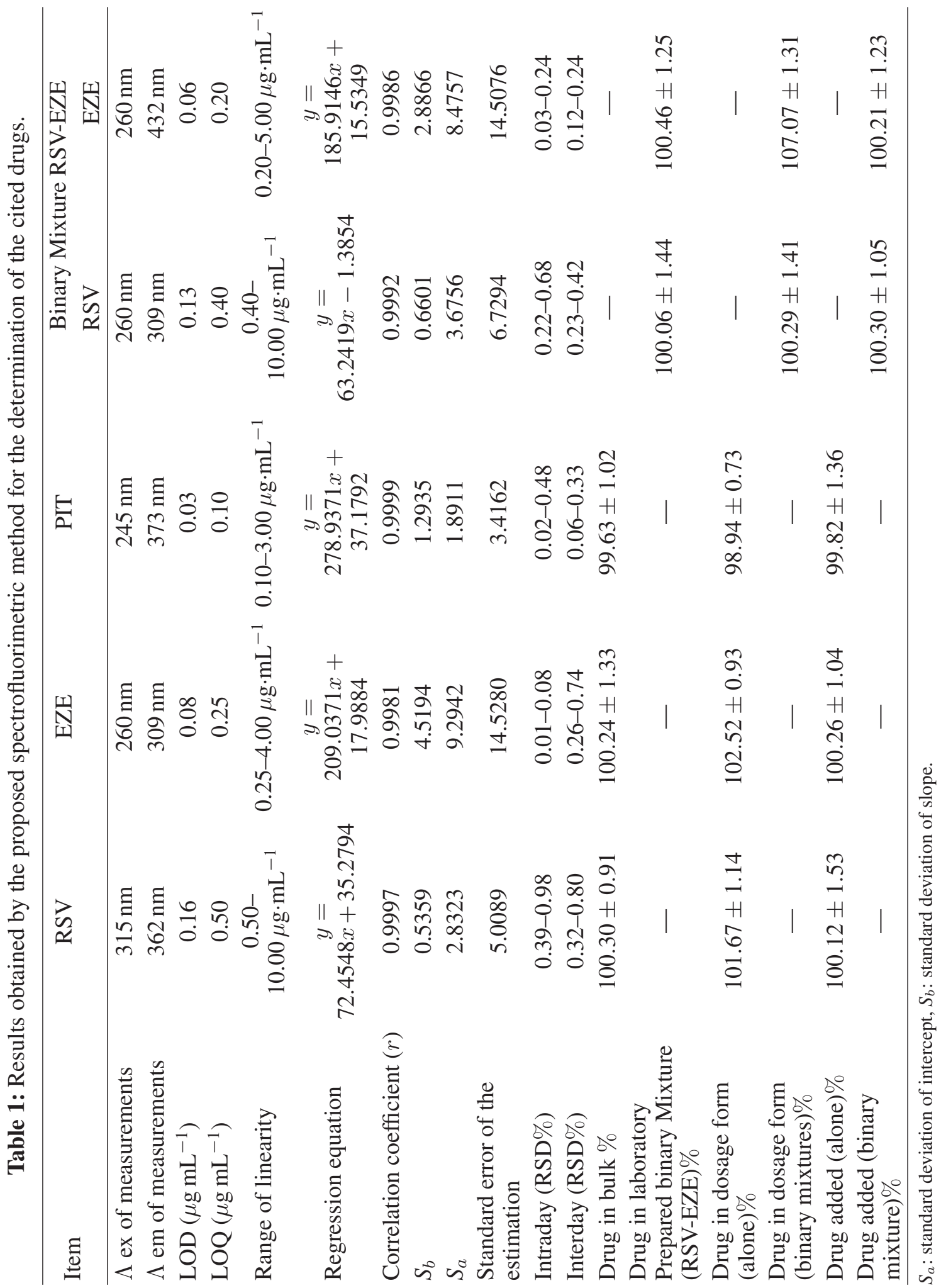




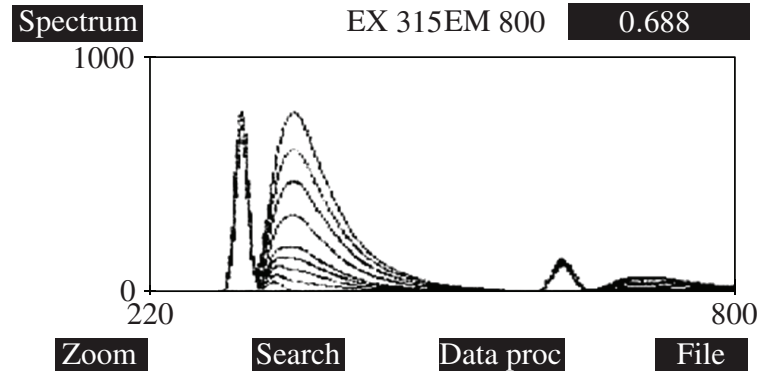

(a)

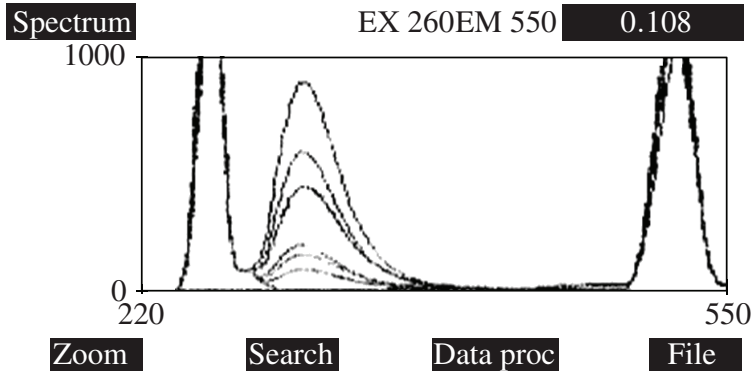

(b)

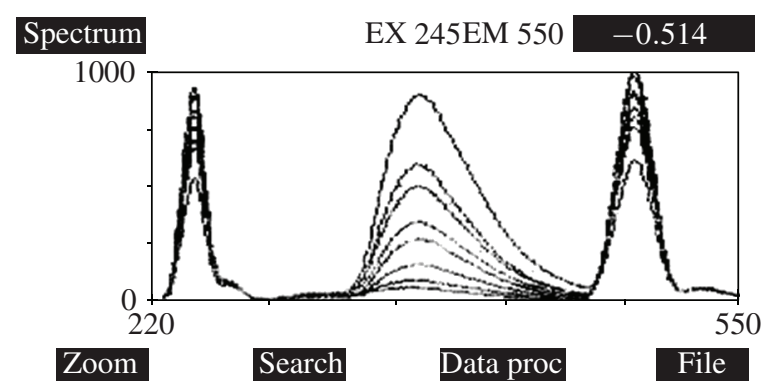

(c)

Figure 2: Fluorescence emission spectrum for rosuvastatin calcium (a), ezetimibe (b), and pitavastatin calcium (c).

spectra and measuring the variation in emission intensity wavelength for a fixed excitation wavelength. Determination for RSV, EZE, and PIT is done at emission wavelengths $362 \mathrm{~nm}, 309 \mathrm{~nm}$, and $373 \mathrm{~nm}$, respectively, at their selected excitation wavelengths. The fluorescence emission spectra of RSV, EZE, and PIT are shown in Figure 2. Methanol was found to be suitable solvent with lowest background noise at the selected excitation and emission wavelengths for RSV and PIT, giving satisfactory results at linearity range of $0.5-10.0 \mu \mathrm{g} \mathrm{mL}^{-1}$ and $0.1-3.0 \mu \mathrm{g} \mathrm{mL}^{-1}$, respectively. While for EZE, acetonitrile was found to give better sensitivity and lower background noise at the selected excitation and emission wavelengths, giving satisfactory results at linearity range of $0.25-4.0 \mu \mathrm{g} \mathrm{mL}^{-1}$.

\subsubsection{For the Binary Mixture RSV-EZE}

Scanning for suitable excitation wavelength for both RSV and EZE was done. Both RSV and EZE were found to be excited at $\lambda$ ex $260 \mathrm{~nm}$, with optimum nonoverlapping emission spectra when RSV and EZE are present in binary mixture solution. Determination of EZE in the binary mixture was thus done by measuring the variation in emission intensity at $\lambda \mathrm{em} 309 \mathrm{~nm}$, while determination of RSV in the binary mixture also was done by measuring the variation in emission intensity at $\lambda \mathrm{em} 432 \mathrm{~nm}$, when applying the suitable excitation wavelength for both EZE and RSV in their binary mixture ( $\lambda$ ex $260 \mathrm{~nm})$. The binary mixture RSV-EZE is found to be of higher sensitivity and lowest background noise when dissolved in acetonitrile. Satisfactory results for linearity range and reproducibility in concentration range from 0.4 to $10.0 \mu \mathrm{g} \mathrm{mL}^{-1}$ for $\mathrm{RSV}$ and 0.2 to $5.0 \mu \mathrm{g} \mathrm{mL}^{1}$ for EZE, in their laboratory-prepared 


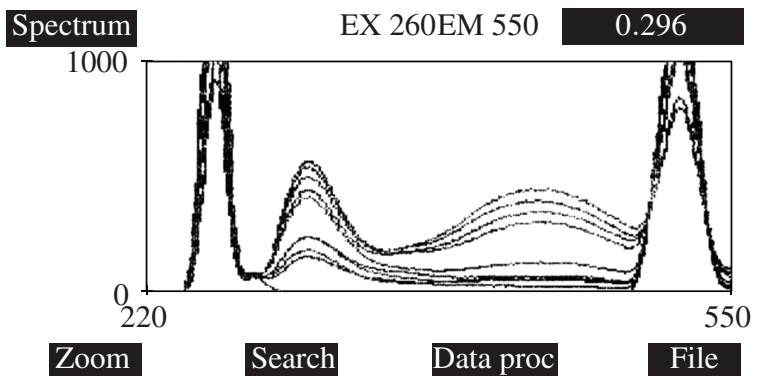

Figure 3: Fluorescence emission spectrum for the laboratory-prepared binary mixture RSV-EZE.

binary mixture. The fluorescence emission spectrum of laboratory-prepared binary mixture RSV-EZE is shown in Figure 3.

\subsection{Method Validation}

\subsubsection{Linearity}

Linearity was studied for the three cited drugs RSV, EZE, and PIT also for the binary mixture RSV-EZE. In this study, 6 to 8 concentrations for each drug were used. A linear relationship between fluorescence intensity and component concentration (c) was obtained. Range of linearity and regression equation $y=b c \pm$ a for each drug is mentioned in Table 1 . The linearity of the calibration curves was validated by the high values of correlation coefficients, as shown in Table 1. The analytical data of the calibration curves including standard deviations for the slope and intercept $\left(S_{b}, S_{a}\right)$ are also summarized in Table 1.

\subsubsection{Accuracy}

Accuracy of the results was calculated by \% recovery of 6 different concentrations of RSV, EZE and 8 different concentrations of PIT in bulk, and also 8 different concentrations of the laboratory prepared mixtures of RSV-EZE and also by standard addition technique applied for the pharmaceutical preparations, all carried out in triplicates. The results obtained including the mean of the recovery, standard deviation, and relative standard deviation are displayed in Table 1.

\subsubsection{Precision}

Precision was estimated by repeatability. The repeatability was assessed by analyzing a solution of $5.0 \mu \mathrm{g} \mathrm{mL}^{-1}$ of RSV and $2.0 \mu \mathrm{g} \mathrm{mL}^{-1}$ of EZE and of PIT $(n=6)$. For the binary mixture RSVEZE, a solution containing $1.0 \mu \mathrm{g} \mathrm{mL}^{-1}$ of both RSV and EZE was analyzed $(n=6)$. The values of the precision (\%R.S.D) of repeatability along with intraday and interday precision (using 3 different concentrations in triplicates for three consecutive days) for the three drugs are displayed in Table 1. 
Table 2: Statistical comparison between the recovery results of the proposed spectrofluorimetric methods and the reference methods for the cited drugs.

\begin{tabular}{lccccc}
\hline Drug & Group & Mean & S.D & $t$-value & $P$-value \\
\hline \multirow{2}{*}{ EZE } & Proposed method & 100.24 & \pm 1.33 & 0.63 & 0.541 \\
& Reference method & 99.86 & \pm 0.94 & & \\
\hline \multirow{2}{*}{ PIT } & Proposed method & 100.30 & \pm 0.91 & \multirow{2}{*}{1.16} & 0.271 \\
& Reference method & 99.68 & \pm 1.03 & & \multirow{2}{*}{0.106} \\
\multirow{2}{*}{ RSV } & Proposed method & 100.30 & \pm 0.91 & \multirow{2}{*}{1.75} & \\
& Reference method & 99.27 & \pm 1.19 & & \\
\hline
\end{tabular}

There is no significant difference between the proposed method and the reference method by using the independent $t$-test at $P<0.05$.

\subsubsection{Specificity}

Specificity is the ability of the analytical method to measure the analyte response in the presence of interferences. In the present work, the spectra of the samples were checked for the appearance of any new spectra of the excipients. No interference from any of the excipients was found at the excitation/emission wavelengths of the examined drugs. In addition, the spectrum of each drug in the sample solution is identical to the spectrum received by the standard solution at the wavelengths applied. Besides, good recoveries were obtained for the samples. These results demonstrate that there was no interference from other materials in the pharmaceutical formulations and therefore confirm the specificity of the method.

\subsubsection{Limit of Detection and Limit of Quantification}

Limit of detection (LOD) which represents the concentration of analyte at S/N ratio of 3 and limit of quantification (LOQ) at which $\mathrm{S} / \mathrm{N}$ is 10 were determined for the proposed method, and results are given in Table 1. LOD and LOQ were computed based on the standard deviation of the response and the slope.

\subsection{Statistical Analysis}

A statistical analysis of the results obtained by the proposed spectroflourometric methods for the determination of (RSV, EZE, and PIT) and those obtained by reported methods was carried out by "SPSS statistical package version 11." The significant difference between groups was tested by one-way ANOVA, as shown in Table 2. The test ascertained that there was no significant difference among the methods.

\section{Conclusion}

The proposed spectrofluorometric methods have the advantages of simplicity, precision, accuracy, and convenience for the determination of the cited antihyperlipidemic drugs: RSV, EZE, and PIT. Besides, 
simultaneous determination of the binary mixture RSV-EZE was carried out. Hence, the proposed methods can be used for the quality control of the cited drugs in ordinary laboratories.

\section{References}

[1] P. Beringer, Remington: The Science and Practice of Pharmacy, Lippincott Williams \& Wilkins, New York, NY, USA, 21st edition, 2006.

[2] H. Lennernäs and G. Fager, "Pharmacodynamics and pharmacokinetics of the HMG-CoA reductase inhibitors. Similarities and differences," Clinical Pharmacokinetics, vol. 32, no. 5, pp. 403-425, 1997.

[3] E. Leitersdorf, "Selective cholesterol absorption inhibition: a novel strategy in lipid-lowering management," International Journal of Clinical Practice, vol. 56, no. 2, pp. 116-119, 2002.

[4] M. V. Heek, C. Farley, D. S. Compton, L. Hoos, and H. R. Davis, "Ezetimibe selectively inhibits intestinal cholesterol absorption in rodents in the presence and absence of exocrine pancreatic function," British Journal of Pharmacology, vol. 134, no. 2, pp. 409-417, 2001.

[5] T. R. Kumar, N. R. Shitut, P. K. Kumar et al., "Determination of rosuvastatin in rat plasma by HPLC: validation and its application to pharmacokinetic studies," Biomedical Chromatography, vol. 20, no. 9, pp. 881-887, 2006.

[6] S. Vittal, T. R. Kumar, N. R. Shitut, M. C. Vinu, R. Muliangi, and N. R. Srinivas, "Simultaneous quantitation of rosuvastatin and gemfibrozil in human plasma by high-performance liquid chromatography and its application to a pharmacokinetic study," Biomedical Chromatography, vol. 20, no. 11, pp. 1252-1259, 2006.

[7] T. N. Mehta, A. K. Patel, G. M. Kulkarini, and G. Suubbaiah, "Determination of rosuvastatin in the presence of its degradation products by a stability-indicating LC method," Journal of AOAC International, vol. 88, no. 4, pp. 1142-1147, 2005.

[8] B. Uyar, M. Celebier, and S. Altinoz, "Spectrophotometric determination of rosuvastatin calcium in tablets," Pharmazie, vol. 62, no. 6, pp. 411-413, 2007.

[9] R. T. Sane, S. S. Kamat, S. N. Menon, S. R. Inamdar, and M. R. Mote, "Determination of rosuvastatin calcium in its bulk drug and pharmaceutical preparations by high-performance thinlayer chromatography," Journal of Planar Chromatography, vol. 18, no. 103, pp. 194-198, 2005.

[10] R. Sistla, V. S. S. K. Tata, Y. V. Kashyap, D. Chandrasekar, and P. V. Diwan, "Development and validation of a reversed-phase HPLC method for the determination of ezetimibe in pharmaceutical dosage forms," Journal of Pharmaceutical and Biomedical Analysis, vol. 39, no. 3-4, pp. 517-522, 2005 .

[11] S. K. Akmar, L. Kothapalli, A. Thomas, S. Jangam, and A. Deshpande, "Reverse phase high performance liquid chromatography method for estimation of ezetimibe in bulk and pharmaceutical formulations," Indian Journal of Pharmaceutical Sciences, vol. 69, no. 5, pp. 695-697, 2007.

[12] S. Singh, B. Singh, R. Bahuguna, L. Wadhwa, and R. Saxena, "Stress degradation studies on ezetimibe and development of a validated stability-indicating HPLC assay," Journal of Pharmaceutical and Biomedical Analysis, vol. 41, no. 3, pp. 1037-1040, 2006.

[13] A. S. Doshi, P. K. Kachhadia, and H. S. Joshi, "Validation of a stability-indicating LC method for assay of ezetimibe in tablets and for determination of content uniformity," Chromatographia, vol. 67, no. 1-2, pp. 137-142, 2008.

[14] S. Oswald, E. Scheuch, I. Cascorbi, and W. Siegmund, "A LC-MS/MS method to quantify the novel cholesterol lowering drug ezetimibe in human serum, urine and feces in healthy subjects genotyped for SLCO1B1," Journal of Chromatography B, vol. 830, no. 1, pp. 143-150, 2006. 
[15] S. Li, G. Liu, J. Jia, X. Li, and C. Yu, "Liquid chromatography-negative ion electrospray tandem mass spectrometry method for the quantification of ezetimibe in human plasma," Journal of Pharmaceutical and Biomedical Analysis, vol. 40, no. 4, pp. 987-992, 2006.

[16] N. Jain, R. Jain, H. Swami, S. Pandey, and D. K. Jain, "Spectrophotometric method for simultaneous estimation of simvastatin and ezetimibe in bulk drug and its combined dosage form," International Journal of Pharmacy and Pharmaceutical Sciences, no. 1, pp. 170-175, 2009.

[17] B. G. Chaudhari, N. Patel, P. B. Shah, L. J. Patel, and V. P. Patel, "Stability-indicating reversedphase liquid chromatographic method for simultaneous determination of atorvastatin and ezetimibe from their combination drug products," Journal of AOAC International, vol. 90, no. 6, pp. 15391546, 2007.

[18] B. G. Chaudhari, N. M. Patel, and P. B. Shah, "Stability-indicating reversed-phase liquid chromatographic method for simultaneous determination of simvastatin and ezetimibe from their combination drug products," Journal of AOAC International, vol. 90, no. 5, pp. 1242-1249, 2007.

[19] H. J. Panchal, B. N. Suhagia, M. M. Patel, and B. H. Patel, "Estimation of pravastatin calcium in tablet dosage forms by column liquid chromatography and ultraviolet spectrophotometry," Journal of AOAC International, vol. 92, no. 1, pp. 158-164, 2009.

[20] S. N. Kumar and J. Baghyalakshmi, "Determination and quantification of pitavastatin calcium in tablet dosage formulation by HPTLC method," Analytical Letters, vol. 40, no. 14, pp. 2625-2632, 2007.

[21] J. H. Shen-Tu, X. Xu, J. Liu et al., "Determination of pitavastatin in human plasma by LC-MS-MS," Chromatographia, vol. 69, no. 9-10, pp. 1041-1047, 2009.

[22] B. Di, M. X. Su, F. Yu et al., "Solid-phase extraction and liquid chromatography/tandem mass spectrometry assay for the determination of pitavastatin in human plasma and urine for application to Phase I clinical pharmacokinetic studies," Journal of Chromatography B, vol. 868, no. 1-2, pp. 95-101, 2008.

[23] L. Tian, Y. Huang, Y. Jia, L. Hua, and Y. Li, "Development and validation of a liquid chromatography-tandem mass spectrometric assay for pitavastatin and its lactone in human plasma and urine," Journal of Chromatography B, vol. 865, no. 1-2, pp. 127-132, 2008.

[24] J. W. Deng, K. B. Kim, I. S. Song et al., "Determination of two HMG-CoA reductase inhibitors, pravastatin and pitavastatin, in plasma samples using liquid chromatography-tandem mass spectrometry for pharmaceutical study," Biomedical Chromatography, vol. 22, no. 2, pp. 131135, 2008.

[25] I. A. Shehata, S. M. EL-Ashry, M. A. EL-Sherbeny, D. T. EL-Sherbeny, and F. Belal, "Fluorimetric determination of some thioxanthene derivatives in dosage forms and biological fluids," Journal of Pharmaceutical and Biomedical Analysis, vol. 22, no. 5, pp. 729-737, 2000.

[26] M. Rizk, F. Belal, F. Ibrahim, S. Ahmed, and N. El-Enany, "Spectrofluorimetric analysis of certain 4-quinolone in pharmaceuticals and biological fluids," Pharmaceutica Acta Helvetiae, vol. 74, no. 4, pp. 371-377, 2000.

[27] F. Belal, F. Ibrahim, S. M. Hassan, and F. A. Aly, "Spectrofluorimetric determination of some pharmaceutically important thioxanthene derivatives," Analytica Chimica Acta, vol. 255, no. 1, pp. 103-106, 1991.

[28] L. I. Bebawy, S. S. Abbas, L. A. Fattah, and H. H. Refaat, "Application of first-derivative, ratio derivative spectrophotometry, TLC-densitometry and spectrofluorimetry for the simultaneous determination of telmisartan and hydrochlorothiazide in pharmaceutical dosage forms and plasma," Farmaco, vol. 60, no. 10, pp. 859-867, 2005. 


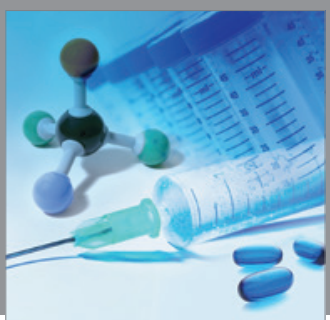

International Journal of

Medicinal Chemistry

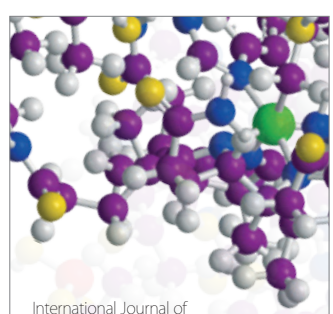

Carbohydrate Chemistry

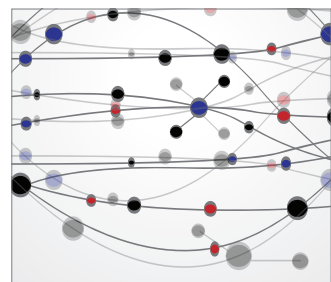

The Scientific World Journal
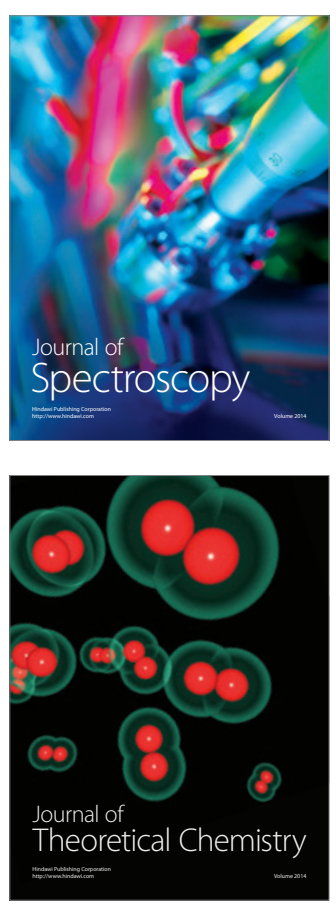
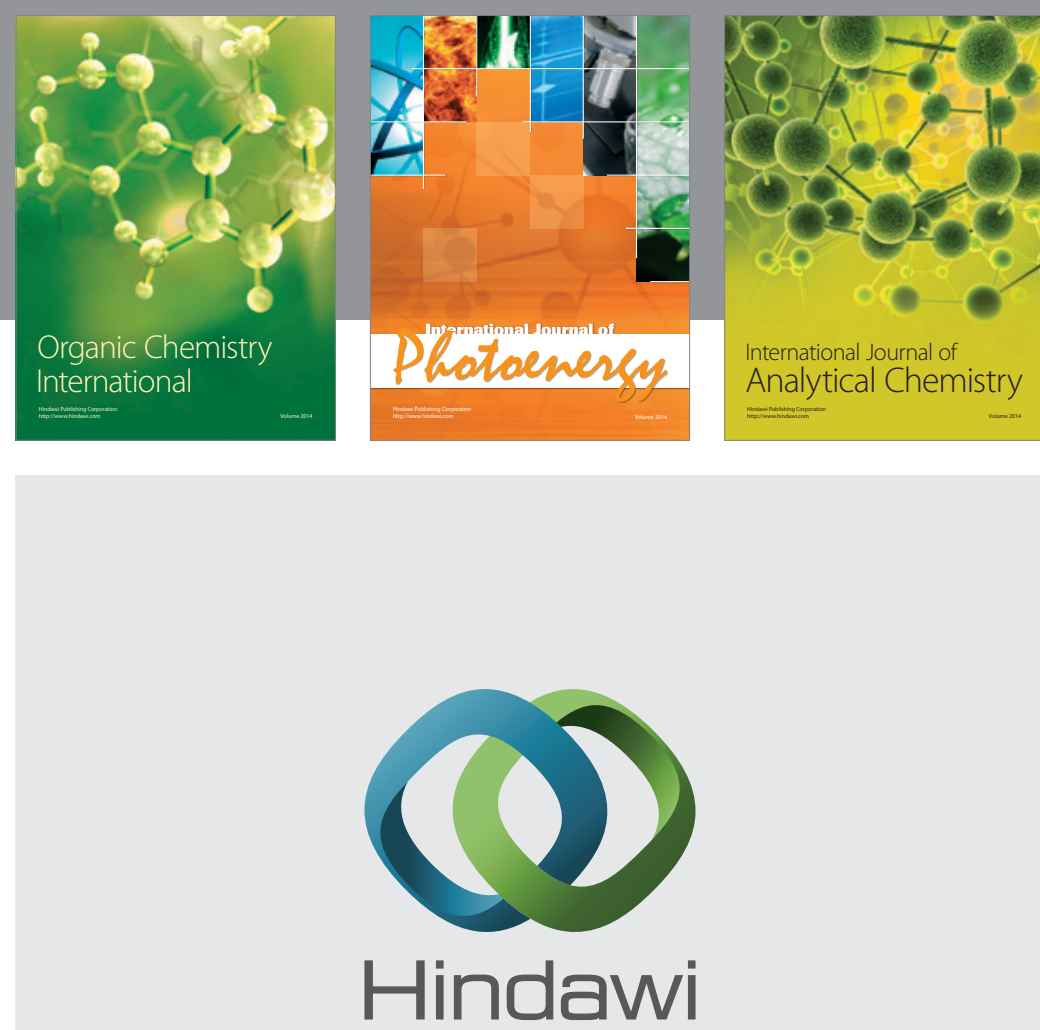

Submit your manuscripts at

http://www.hindawi.com
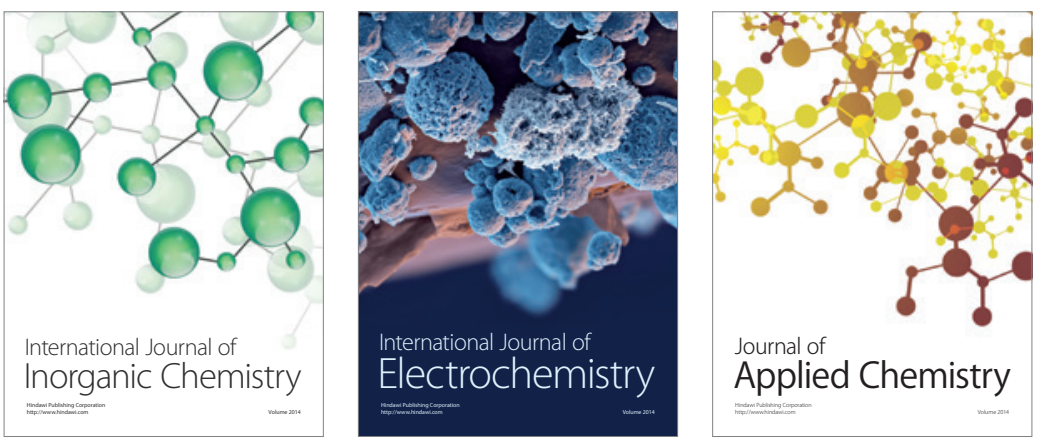

Journal of

Applied Chemistry
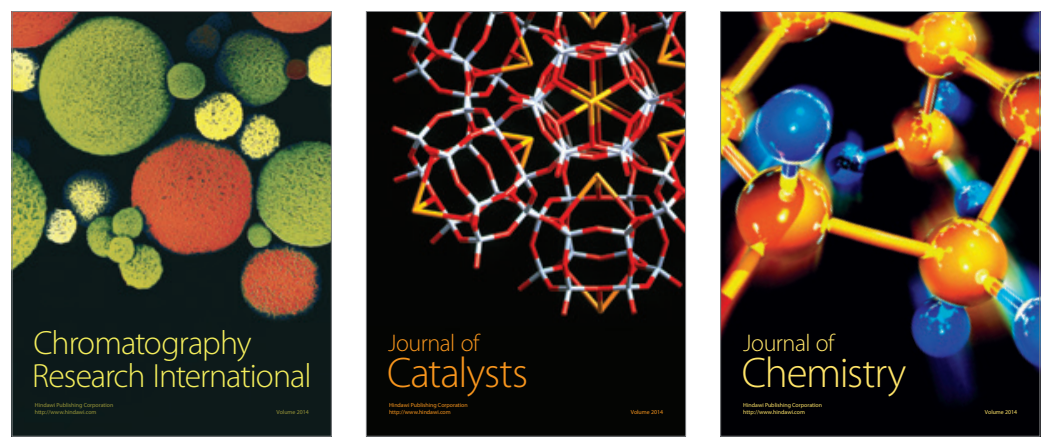
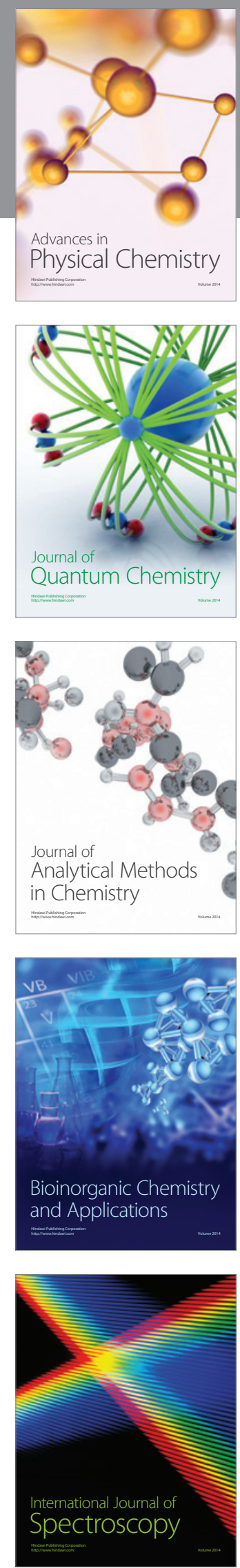